\title{
Sliding Mode Control, with Integrator, for a Class of MIMO Nonlinear Systems
}

\author{
Anouar Benamor ${ }^{1}$, Larbi Chrifi-alaui ${ }^{2}$, Hassani Messaoud ${ }^{1}$, Mohamed Chaabane ${ }^{3}$ \\ ${ }^{1}$ Unit'e de Recherche en Automatique, Traitement du Signal et Image de l'ENIM, ATSI-ENIM, Monastir, Tunisie \\ ${ }^{2}$ Laboratoire des Technologies Innovantes, Université de Picardie Jules Verne, Mitterrand, France \\ ${ }^{3}$ Unité de Commande Automatique, l'Ecole Nationale d'Ingénieurs de Sfax, Sfax, Tunisie \\ E-mail:anouar.benamor@yahoo.fr,hassani.messaoud@enim.rnu.tn,larbi.alaoui@u-picardie.fr, \\ chaabaneuca@yahoo.fr \\ Received January 24, 2011; revised March 8, 2011; accepted March 22, 2011
}

\begin{abstract}
In this paper, the robust control problem of general nonlinear multi-input multi-output (MIMO) systems is proposed. The robustness against unknown disturbances is considered. Two algorithms based on the Sliding Mode Control (SMC) for nonlinear coupled Multi-Input Multi-Output (MIMO) systems are proposed: the first order sliding mode control (FOSMC) with saturation (sat) function and the FOSMC with sat combined with integrator controller. Those algorithms were simulated and implemented on the three tanks test-bed system and the experimental results confirm the effectiveness of our control design.
\end{abstract}

Keywords: Sliding Mode Control, Integrator, Nonlinear Systems, Coupled, Mimo, Uncertain, Liquid Level Control

\section{Introduction}

The SMC is a widely used approach to design robust control law of uncertain systems. The advantage of such approach is its robustness to parameter variations and disturbances $[1,2]$. But the major inconvenience of classic sliding mode control is the existence of chattering phenomenon [3], which may induce many undesirable oscillations in control signal. Some attempts on chattering [4] canceling have considered continuous functions instead of sign one. However the provided results lead to a large steady state error which can be reduced using the integral action [5-7]. Moreover even though there exist many works dealing with sliding mode control in the case of Single Input Single Output (SISO) systems [8], there is lock of results when the addressed process is Multi-Input Multi-Output (MIMO) one. This shortage is due to output coupling problem.

In this paper, we propose a first order sliding mode control using Sat function and this control combined with an integrator corrector. Experimental results, operated on a three tank system, are presented to illustrate the effectiveness of the proposed controllers.

The paper is organized as follows. In Section 2 we remind the classical sliding mode control of coupled MIMO nonlinear systems and its robustness to parameter uncertainties and external disturbances. Section three is devoted to SMC with sat function and integral action. The model of the coupled three tanks system and its control is presented in Section 4. The simulation and experimental results are presented in Sections 5 and 6. Finally Section 7 concludes the paper.

\section{Sliding Mode Control}

Consider a MIMO non linear system with $p$ inputs and $m$ outputs defined by the following state representation:

$$
\left\{\begin{array}{l}
\dot{x}=f(x, t)+g(x, t) u \\
y=c(x, t)
\end{array}\right.
$$

where $x$ is the n-dimensional state vector and $y$ is the $\mathrm{m}$-dimensional output vector.

$x=\left[\begin{array}{ccc}x_{1} & \cdots & x_{n}\end{array}\right]^{T}$ and $y=\left[\begin{array}{lll}y_{1} & \cdots & y_{m}\end{array}\right]^{T}$ is the $\mathrm{m}$-dimensional vector, the coefficients of which are nonlinear functions $c_{i}(x, t), f(x, t)$ is the n-dimensional vector, the coefficients of which are nonlinear functions $f_{i}(x, t), g(x, t)$ is a $(n \times p)$ matrix with coefficients are the nonlinear functions $g_{i j}(x, t)$ and $u$ is the p-dimensional control vector of coefficients $u_{i}$. 


$$
u=\left[\begin{array}{lll}
u_{1} & \cdots & u_{p}
\end{array}\right]^{T}
$$

\subsection{Classical Sliding Mode Control}

Consider the sliding surface [9] defined by:

$$
s=\left[\begin{array}{lll}
s_{1} & \cdots & s_{p}
\end{array}\right]^{T}
$$

where:

$$
s_{i}=\sum_{k=0}^{r_{i}-1} \lambda_{k}^{(i)} e_{i}^{(k)}, \text { for } i=1, \cdots, p
$$

with:

$r_{i}$ is the relative degree of the error $e_{i} \quad \lambda_{r_{-1}}^{(i)}=1$ and for $\mathrm{k}=1, \cdots, \mathrm{r}_{\mathrm{i}}-2, \lambda_{k}^{(i)}$ are constants chosen so that $\lambda_{0}^{(i)}+\lambda_{1}^{(i)} p+\cdots+\lambda_{r_{i-1}}^{(i)} p^{r_{i-1}}$ is a Hurwitz polynomial one and $e_{i}^{(k)}$ is the $k^{\text {th }}$ order derivative of the error $e_{i}$.

$$
e_{i}=y_{i}-y_{i}^{d}, \quad i=1, \cdots, r_{i}-1 .
$$

where $y_{i}^{d}$ is the desired output. The derivative of $s_{i}$ is:

$$
\frac{\mathrm{d} s_{i}}{\mathrm{~d} t}=\sum_{k=0}^{r_{i}-1} \lambda_{k}^{(i)}\left(\frac{\partial \mathrm{e}_{i}}{\partial t}+\sum_{j=1}^{n} \frac{\partial \mathrm{e}_{i}}{\partial x_{j}} \dot{x}_{j}\right)
$$

Replacing $x_{j}$ by its expression in (1) and omitting the index $(\mathrm{x}, \mathrm{t})$, relation $(6)$ becomes.

$$
\frac{\mathrm{d} s_{i}}{\mathrm{~d} t}=\sum_{k=0}^{r_{i}-1} \lambda_{k}^{(i)}\left(\frac{\partial e_{i}}{\partial t}+\sum_{j=1}^{n} \frac{\partial e_{i}}{\partial x_{j}} f_{j}+\sum_{l=1}^{p} \sum_{j=1}^{n} \frac{\partial e_{i}}{\partial x_{j}} g_{j l} u_{l}\right)
$$

which can be written as:

$$
\frac{\mathrm{d} s_{i}}{\mathrm{~d} t}=h_{i}+b_{i 1} u_{1}+\cdots+b_{i P} u_{P}=h_{i}+\sum_{l=1}^{p} b_{i l} u_{l}
$$

with:

$$
\begin{aligned}
& h_{i}=\sum_{k=0}^{r_{i}-1} \lambda_{k}^{(i)}\left(\frac{\partial s_{i}}{\partial t}+\sum_{j=1}^{n} \frac{\partial s_{i}}{\partial x_{j}} f_{i}\right) \text { and } \\
& b_{i k}=\sum_{k=0}^{r_{i}-1} \lambda_{k}^{(i)}\left(\sum_{j=1}^{n} \frac{\partial s_{i}}{\partial x_{j}} g_{j l}\right)
\end{aligned}
$$

Then we can write the derivative of the surface vector as

$$
\dot{s}=h+b u
$$

with:

$$
h=\left[\begin{array}{c}
h_{1} \\
\vdots \\
h_{P}
\end{array}\right] \text { and } b=\left[\begin{array}{ccc}
b_{11} & \cdots & b_{1 P} \\
\vdots & \ddots & \vdots \\
b_{P 1} & \cdots & b_{P P}
\end{array}\right]
$$

\section{Theorem 1.}

The control law for the first order sliding mode control (FOSMC) of the system 1 so that the sliding surfaces go to zero in a finite time is defined by:

$$
u=-b^{-1}\left(h+\left[\begin{array}{c}
k_{1} \operatorname{sign}\left(s_{1}\right) \\
\vdots \\
k_{P} \operatorname{sign}\left(s_{P}\right)
\end{array}\right]\right)
$$

with $k_{i}$ a positive constant and $b$ an invertible matrix.

Proof.

Consider the following Lyapunov function:

$$
V=\frac{1}{2} s^{T} s=\frac{1}{2}\left(s_{1}^{2}+\cdots+s_{P}^{2}\right)
$$

the derivative of $V$ is:

$$
\dot{V}=s_{1} \dot{s}_{1}+\cdots+s_{P} \dot{s}_{P}=s^{T} \dot{s}
$$

Using (9) we have:

$$
\dot{V}=s^{T}(h+b u)
$$

Replacing $u$ by its expression (10) in Equation (9), we obtain:

$$
\dot{s}=-\left[\begin{array}{c}
k_{1} \operatorname{sign}\left(s_{1}\right) \\
\vdots \\
k_{P} \operatorname{sign}\left(s_{P}\right)
\end{array}\right]
$$

then

$$
\dot{V}=-s^{T}\left[\begin{array}{c}
k_{1} \operatorname{sign}\left(s_{1}\right) \\
\vdots \\
k_{P} \operatorname{sign}\left(s_{P}\right)
\end{array}\right]=\sum_{i=1}^{p}-k_{i} \operatorname{sign}\left(s_{i}\right) s_{i}=-\sum_{i=1}^{P} k_{i}\left|s_{i}\right| \leq 0
$$

Since $k_{i}(i=1, \cdots, p)$ are positive we have $V<0$.

Then, the Lyapunov function $V$ tends to 0 and therefore all surfaces $s_{i}$ tend to zero, hence the existence of the first order sliding mode.

To prove the finite time convergence of our control we take the Equation (14), we have $\dot{s}_{i}=-k_{i} \operatorname{sign}\left(s_{i}\right) s_{i}$ then $s_{i} \dot{s}_{i}=-k_{i}\left|s_{i}\right| \leq-\mu_{i}\left|s_{i}\right|$, with $0 \prec \mu \leq k_{i}$, which is the $\mu$ reachability condition [10], then the finite time convergence.

\subsection{Robustness to Parametric Uncertainties and External Disturbances}

Consider an uncertain MIMO nonlinear system:

$$
\dot{x}=\hat{f}(x, t)+\Delta f(x, t)+(\hat{g}(x, t)+\Delta g(x, t)) u+d
$$

where $x \in \mathfrak{R}^{n}$ is the state vector, $u \in \mathfrak{R}^{p}$ is the inputcontrol bounded as $\left|u_{i}\right| \leq u_{i \max }$ for $i=1$ to $\mathrm{p}$, the vector field $f(x, t)=\hat{f}(x, t)+\Delta f(x, t)$ is continuous and smooth, where $\hat{f}(x, t)$ is the nominal part and $\Delta f(x, t)$ is the uncertain part bounded by a known function. $d \in D \subset \mathfrak{R}^{n}$ represents the disturbances. The dynamic $g(t, x)$ is not exactly known and it is written as the sum of the nominal part $\hat{g}(x, t)$ and the uncertain part $\Delta g(x, t)$. 
with:

$$
\begin{gathered}
\hat{f}(x, t)=\left[\begin{array}{c}
\hat{f}_{1}(x, t) \\
\vdots \\
\hat{f}_{n}(x, t)
\end{array}\right], \Delta \hat{f}(x, t)=\left[\begin{array}{c}
\Delta \hat{f}_{1}(x, t) \\
\vdots \\
\Delta \hat{f}_{n}(x, t)
\end{array}\right], d=\left[\begin{array}{c}
d_{1} \\
\vdots \\
d_{n}
\end{array}\right] \\
\hat{g}(x, t)=\left[\begin{array}{ccc}
\hat{g}_{11}(x, t) & \cdots & \hat{g}_{1 P}(x, t) \\
\vdots & \ddots & \vdots \\
\hat{g}_{n 1}(x, t) & \cdots & \hat{g}_{n P}(x, t)
\end{array}\right] \\
\text { and } \Delta g(x, t)=\left[\begin{array}{ccc}
\Delta g_{11}(x, t) & \cdots & \Delta g_{1 P}(x, t) \\
\vdots & \ddots & \vdots \\
\Delta g_{n 1}(x, t) & \cdots & \Delta g_{n P}(x, t)
\end{array}\right]
\end{gathered}
$$

Then the derivative of the sliding surface takes the following form:

$$
\frac{\mathrm{d} s_{i}}{\mathrm{~d} t}=\hat{h}_{i}+\Delta h_{i}+\sum_{k=1}^{p}\left(\hat{b}_{i k}+\Delta b_{i k}\right) u_{k}+\delta_{i}
$$

then:

$$
\begin{gathered}
\dot{s}=\hat{h}+\Delta h+(\hat{b}+\Delta b) u+\delta \\
\hat{h}(x, t)=\left[\begin{array}{c}
\hat{h}_{1}(x, t) \\
\vdots \\
\hat{h}_{p}(x, t)
\end{array}\right], \Delta h(x, t)=\left[\begin{array}{c}
\Delta h_{1}(x, t) \\
\vdots \\
\Delta h_{p}(x, t)
\end{array}\right], \delta=\left[\begin{array}{c}
\delta_{1} \\
\vdots \\
\delta_{p}
\end{array}\right] \\
\hat{b}(x, t)=\left[\begin{array}{ccc}
\hat{b}_{11}(x, t) & \cdots & \hat{b}_{1 p}(x, t) \\
\vdots & \ddots & \vdots \\
\hat{b}_{p 1}(x, t) & \cdots & \hat{b}_{p p}(x, t)
\end{array}\right] \\
\text { and } \Delta b(x, t)=\left[\begin{array}{cccc}
\Delta b_{11}(x, t) & \cdots & \Delta b_{1 p}(x, t) \\
\vdots & \ddots & \vdots \\
\Delta b_{p 1}(x, t) & \cdots & \Delta b_{p p}(x, t)
\end{array}\right]
\end{gathered}
$$

\section{Theorem 2.}

Consider the uncertain system defined by Equation (16). The control law:

$$
u=-\hat{b}^{-1}\left(\hat{h}+\left[\begin{array}{c}
k_{1} \operatorname{sign}\left(s_{1}\right) \\
\vdots \\
k_{P} \operatorname{sign}\left(s_{P}\right)
\end{array}\right]\right)
$$

with $k_{i}$ satisfying:

$$
k_{i}>\alpha_{i}+\delta_{i}^{*}+\sum_{k=1}^{p} \beta_{i k} u_{k \max }
$$

and where $\alpha_{i}, \beta_{i k}, \delta_{i}^{*}$ and $u_{k \max }$ are the upper bounds of uncertainties, $\Delta h_{1}, \Delta b_{i k}, \delta_{i}$ and $u_{k}$ respectively, ensures the finite time convergence of the sliding surface to zero.

\section{Proof.}

The expression of the derivative of the surface becomes:

$$
\dot{s}=\hat{h}+\Delta h+(\hat{b}+\Delta b) u+\delta
$$

where $\delta$ is the p-dimensional vector whose coefficients are :

$$
\delta_{i}=\sum_{j=1}^{n} \frac{\partial s_{i}}{\partial x_{j}} \mathrm{~d}_{j} \text { for } i=1 \text { to } p
$$

Using the control expression in (18) we have:

$$
\dot{s}=-\left[\begin{array}{c}
k_{1} \operatorname{sign}\left(s_{1}\right) \\
\vdots \\
k_{P} \operatorname{sign}\left(s_{P}\right)
\end{array}\right]+\Delta h+\Delta b u+\delta
$$

The derivative of the surface $s_{i}$ is then written:

$$
\dot{s}_{i}=\Delta h_{i}-k_{i} \operatorname{sign}\left(s_{i}\right)+\sum_{k=1}^{p} \Delta b_{i k} u_{k}+\delta_{i}
$$

and the derivative of the Lyapunov function given by (12) is: $\dot{V}=\sum_{i=1}^{n} s_{i} \dot{s}_{i}$

$\dot{V}$ will be negative if the following conditions are satisfied: $s_{i} \dot{s}_{i} \leq 0$ for $i=1$ to $\mathrm{p}$.

If $\mathrm{s}_{i}>0$ then $\dot{s}_{i}>0$, we have:

$$
\Delta h_{i}-k_{i}+\sum_{k=1}^{p} \Delta b_{i k} u_{k}+\delta_{i}<0
$$

then

$$
\Delta h_{i}+\sum_{k=1}^{p} \Delta b_{i k} u_{k}+\delta_{i}<k_{i}
$$

If $s_{i}<0$ then $\dot{s}_{i}<0$, we have:

$$
\Delta h_{i}+k_{i}+\sum_{k=1}^{p} \Delta b_{i k} u_{k}+\delta_{i}>0
$$

then

$$
-\left(\Delta h_{i}+\sum_{k=1}^{p} \Delta b_{i k} u_{k}+\delta_{i}\right)<k_{i}
$$

The conditions (24) and (26) are satisfied if:

$$
\alpha_{i}+\sum_{k=1}^{p} \beta_{i k} u_{k \max }+\delta_{i}^{*}<k_{i}
$$

then

$$
\dot{V}<0
$$

\section{SMC with Sat}

The major inconvenient of classic sliding mode control is the existence of chattering phenomenon. To avoid this 
problem, we approximate the «sign» function by continuous functions such as sat function [9] defined by:

$$
\operatorname{sat}\left(s_{i}\right)= \begin{cases}\operatorname{sign}\left(s_{i}\right) & \text { if }\left|s_{i}\right|>\varphi_{i} \\ \frac{s_{i}}{\varphi_{i}} & \text { if }\left|s_{i}\right| \leq \varphi_{i}\end{cases}
$$

where $\varphi_{i}$ is a positive constant that defines the thickness of the boundary layer.

\section{Theorem 3.}

The control law for the first order sliding mode control (FOSMC) with sat of the system (1) is defined by:

$$
u=-b^{-1}\left(h+\left[\begin{array}{c}
k_{1} s a t\left(s_{1}\right) \\
\vdots \\
k_{P} s a t\left(s_{P}\right)
\end{array}\right]\right)
$$

with $k_{i}$ a positive constant and $b$ an invertible matrix.

Proof.

We consider the same Lyapunov function defined by Equation (11). Its derivative with using control defined in (31) is:

$$
\dot{V}=-s^{T}\left[\begin{array}{c}
k_{1} \operatorname{sat}\left(s_{1}\right) \\
\vdots \\
k_{P} \operatorname{sat}\left(s_{P}\right)
\end{array}\right]
$$

then

$$
\dot{V}=-\sum_{i=1}^{p} k_{i} \operatorname{sat}\left(s_{i}\right) s_{i}
$$

Using sat definition given by (28), we have:

$$
\operatorname{sat}\left(s_{i}\right) s_{i}= \begin{cases}\operatorname{sign}\left(s_{i}\right) s_{i} \geq 0 & \text { if }\left|s_{i}\right|>\varphi_{i} \\ \frac{s_{i}^{2}}{\varphi_{i}} \geq 0 & \text { if }\left|s_{i}\right| \leq \varphi_{i}\end{cases}
$$

Therefore:

$$
\operatorname{sat}\left(s_{i}\right) s_{i} \geq 0
$$

then

$$
\dot{V}<0
$$

\section{Remark.}

In the boundary layer the derivative of the surface is:

$$
s_{i}=\frac{s_{i}}{\varphi_{i}}
$$

then

$$
s_{i}=s_{i}\left(t_{r i}\right) \mathrm{e}^{-\frac{t-t_{r i}}{\varphi_{i}}}=\varphi_{i} \mathrm{e}^{-\frac{t-t_{r i}}{\varphi_{i}}}
$$

with:

$t_{r i}$ is the start time of boundary layer. then

$$
s_{i} \neq 0 \quad \forall t>t_{r i} \text { for } i=1, \cdots, p
$$

In order to solve a steady-state error problem, an integral sliding manifold was proposed in [10]. This development is introduced and justified only by tests on specific systems. Our idea consists on reconstituting a control law to eliminate steady-state error created by disturbance. To do so we added an integral action when the trajectories of states approach their references [11-14].

\section{Proposition.}

Consider the uncertain system defined by Equation (1). The control law FOSMC with integrator to eliminate steady-state error is defined by:

$$
u=-b^{-1}\left(h+\left[\begin{array}{c}
k_{1} \operatorname{sat}\left(s_{1}\right) \\
\vdots \\
k_{P} \operatorname{sat}\left(s_{P}\right)
\end{array}\right]\right)+K\left[\begin{array}{c}
\int\left(y_{1}-y_{1}^{d}\right) \mathrm{d} t \\
\vdots \\
\int\left(y_{1}-y\right)_{1}^{d} \mathrm{~d} t
\end{array}\right]
$$

with

$$
K=\left[\begin{array}{ccc}
K_{11} & \cdots & K_{1 p} \\
\vdots & \ddots & \vdots \\
K_{p 1} & \cdots & K_{p p}
\end{array}\right]
$$
by:

The coefficients $K_{i j}$ are the integrator constant defined

$$
K_{i j}=\left\{\begin{array}{cc}
\text { positive constant } & \text { if }\left|y_{i}-y_{i}^{d}\right|>\varphi_{\mathrm{j}} \\
0 & \text { if }\left|y_{i}-y_{i}^{d}\right| \leq \varphi_{\mathrm{j}}
\end{array}\right.
$$

with $\varphi_{\mathrm{j}}$ a positive constant.

\section{Validation}

\subsection{System Description and Modeling}

The considered process is a three tank system, which have two inputs and three outputs. It consists on three cylindrical tanks with identical Section $a$ supplied with distilled water, which are serially interconnected by two cylindrical pipes of identical Sections $S_{n}$. The pipes of communication between the tanks $T_{1}$ and $T_{2}$ are equipped with manually adjustable valves; the flow rates of the connection pipes can be controlled using ball valves $a_{z 1}$ and $a_{z 2}$. The plant has one outlet pipe located at the bottom of tank $T_{3}$. There are three other pipes each installed at the bottom of each tank; they are provided with a direct connection (outflow rate) to the reservoir with ball valves $b_{z 1}, b_{z 2}$ and $b_{z 3}$, respectively, it can only be manipulated manually. The pumps 1 and 2 are supplied by water from the reservoir with flow rates $Q_{1}(t)$ and $Q_{2}(t)$, respectively. The necessary level measurements $h_{1}(t)$, 
$h_{2}(t)$ and $h_{3}(t)$ are carried out by the piezo-resistive differential pressure sensors.

The state Equations are obtained by writing that the variation of the water volume in a tank is equal to the difference between the incoming flow and the outgoing flows, that means, the water of the tanks 1 and 2 can flow toward the tank 3 .

Then, the system can be represented by the following Equations:

$$
\dot{h}_{i}(t)=\frac{1}{A}\left(Q_{i}^{\text {in }}(t)-Q_{i j}^{\text {out } 1}(t)-Q_{i j}^{\text {out } 2}(t)\right) i, j=1,2,3
$$

where $Q_{i}^{\text {in }}(t)$ is the flow through pump $i(i=1 ; 2)$ and $Q_{i j}^{o u t 1}(t)$ represents the flow rates of water between the tanks $i$ and $j \quad(i, j=1,2,3 \quad \forall i \neq j)$, and can be expressed using the law of Torricelli[15].

$$
Q_{i j}^{o u t 1}(t)=a_{z i} S_{n} \operatorname{sign}\left(h_{i}-h_{j}\right) \sqrt{2 g\left|h_{i}-h_{j}\right|} \quad i, j=1,3
$$

and $Q_{i j}^{\text {out } 2}(t)$ represents the outflow rate, given by:

$$
Q_{i j}^{\text {out } 2}(t)=b_{z j} S_{L} \sqrt{2 g h_{i}} \quad j=1,2,3
$$

where $h_{i}(t), Q_{i}^{\text {in }}(t)$ and $Q_{i j}^{\text {out }}(t)$ are respectively the levels of water, the input flow and the output flow rates.

The parameters of three tank system are defined in the Table 1.

The controlled signals are the water levels $\left(h_{2}, h_{3}\right)$ of tanks 2 and tank 3. These levels are controlled by two pumps. The system can be considered as a multi input multi output system (MIMO) where the input are inflow rates $Q_{1}, Q_{2}$ and the output are liquid levels $h_{2}, h_{3}$. Then the three tank systems can be modeled by the following three differential Equations as shown in (43): where the parameters $c_{i}, i=1,3$ and $B_{j}, j=1,2,3,4$ are defined by:

$$
c_{i}=\frac{1}{A} a_{z i} S_{n} \sqrt{2 g} \quad i=1,3
$$

$$
B_{j}=\frac{1}{A} b_{z j} S_{L} \sqrt{2 g} \quad j=1,2,3,4
$$

While taking $B_{1}=B_{2}=B_{3}=0$, the three Equations of the system become (see Equation (44)):

At equilibrium, for constant water level set point, the level derivatives must be zero.

$$
\dot{h}_{1}=\dot{h}_{2}=\dot{h}_{3}=0
$$

Therefore, using (45) in the steady state, the following algebraic relationship holds.

$$
\left\{\begin{array}{l}
-c_{1} \operatorname{sign}\left(h_{1}-h_{3}\right) \sqrt{\left|h_{1}-h_{3}\right|}+\frac{Q_{1}}{a}=0 \\
c_{1} \operatorname{sign}\left(h_{1}-h_{3}\right) \sqrt{\left|h_{1}-h_{3}\right|}-c_{3} \operatorname{sign}\left(h_{3}-h_{2}\right) \sqrt{\left|h_{3}-h_{2}\right|}=0
\end{array}\right.
$$

For the coupled tanks system, the fluid flow $Q_{1}$ into tank 1 , cannot be negative because the pump can only drive water into the tank, then:

$$
Q_{1} \geq 0
$$

From (47) we have $c_{1} \operatorname{sign}\left(h_{1}-h\right)_{3} \sqrt{\left|h_{1}-h_{3}\right|}=\frac{Q_{1}}{a}$ and $c_{1} \operatorname{sign}\left(h_{1}-h_{3}\right) \sqrt{\left|h_{1}-h_{3}\right|}-c_{3} \operatorname{sign}\left(h_{3}-h_{2}\right) \sqrt{\left|h_{3}-h_{2}\right|}$

Then $\left(h_{1}-h_{3}\right) \geq 0$ and $\left(h_{3}-h_{2}\right) \geq 0$. Therefore if we assume.

$$
x_{1}=h_{1}, x_{2}=h_{2}, x_{3}=h_{3}, u_{1}=Q_{1} \text { and } u_{2}=Q_{2}
$$

We have

$$
\left\{\begin{array}{l}
\dot{x}_{1}=-c_{1} \sqrt{\left|x_{1}-x_{3}\right|}+\frac{u_{1}}{a} \\
\dot{x}_{2}=c_{3} \sqrt{\left|x_{3}-x_{2}\right|}-B_{4} \sqrt{x_{2}}+\frac{u_{2}}{a} \\
\dot{x}_{3}=c_{1} \sqrt{\left|x_{1}-x_{3}\right|}-c_{3} \sqrt{\left|x_{3}-x_{2}\right|}
\end{array}\right.
$$

$$
\begin{aligned}
& \frac{\mathrm{d} h_{1}}{\mathrm{~d} t}=-c_{1} \operatorname{sign}\left(h_{1}-h_{3}\right) \sqrt{\left|h_{1}-h_{3}\right|}-B_{1} \sqrt{h_{1}}+\frac{Q_{1}}{a} \\
& \frac{\mathrm{d} h_{2}}{\mathrm{~d} t}=c_{3} \operatorname{sign}\left(h_{3}-h_{2}\right) \sqrt{\left|h_{3}-h_{2}\right|}-\left(B_{4}+B_{2}\right) \sqrt{h_{2}}+\frac{Q_{2}}{a} \\
& \frac{\mathrm{d} h_{3}}{\mathrm{~d} t}=c_{1} \operatorname{sign}\left(h_{1}-h_{3}\right) \sqrt{\left|h_{1}-h_{3}\right|}-\left(B_{3}+B_{2}\right) \sqrt{h_{3}}-c_{3} \operatorname{sign}\left(h_{3}-h_{2}\right) \sqrt{\left|h_{3}-h_{2}\right|} \\
& \left\{\begin{array}{l}
\frac{\mathrm{d} h_{1}}{d t}=-c_{1} \operatorname{sign}\left(h_{1}-h_{3}\right) \sqrt{\left|h_{1}-h_{3}\right|}+\frac{Q_{1}}{a} \\
\frac{\mathrm{d} h_{2}}{d t}=c_{3} \operatorname{sign}\left(h_{3}-h_{2}\right) \sqrt{\left|h_{3}-h_{2}\right|}-B_{4} \sqrt{h_{2}}+\frac{Q_{2}}{a} \\
\frac{\mathrm{d} h_{3}}{d t}=c_{1} \operatorname{sign}\left(h_{1}-h_{3}\right) \sqrt{\left|h_{1}-h_{3}\right|}-c_{3} \operatorname{sign}\left(h_{3}-h_{2}\right) \sqrt{\left|h_{3}-h_{2}\right|}
\end{array}\right.
\end{aligned}
$$


which can be written in the same form of (1) as:

$$
\left\{\begin{array}{l}
\dot{x}=f(t, x)+g u \\
y=c x
\end{array}\right.
$$

where

$$
\begin{aligned}
& x=\left[\begin{array}{lll}
x_{1} & x_{2} & x_{3}
\end{array}\right]^{T}, u=\left[\begin{array}{ll}
u_{1} & u_{2}
\end{array}\right]^{T}, y=\left[\begin{array}{cc}
x_{2} & x_{3}
\end{array}\right]^{T} \\
& f(t, x)=\left(\begin{array}{c}
-c_{1} \sqrt{x_{1}-x_{3}} \\
c_{3} \sqrt{x_{3}-x}-B_{4} \sqrt{x_{2}} \\
c_{1} \sqrt{x_{1}-x_{3}}-c_{3} \sqrt{x_{3}-x_{2}}
\end{array}\right), g=\left(\begin{array}{cc}
\frac{1}{a} & 0 \\
0 & \frac{1}{a} \\
0 & 0
\end{array}\right) \text { and }
\end{aligned}
$$$$
c=\left(\begin{array}{lll}
0 & 1 & 0 \\
0 & 0 & 1
\end{array}\right)
$$

\subsection{Sliding Mode Control of the Three Tank System}

The objective is to regulate the water levels of tank 2 and tank 3 by using both laws defined in Section 3 .

The vector of the sliding surface is given by:

$$
s=\left[\begin{array}{ll}
s_{1} & s_{2}
\end{array}\right]^{T}
$$

where:

$$
s_{1}=\left(x_{2}-x_{2 d}\right) \text { and } s_{2}=\lambda\left(x_{3}-x_{3 d}\right)+\left(\dot{x}_{3}-\dot{x}_{3 d}\right)
$$

$x_{2 d}$ and $x_{3 d}$ are the desired water levels of tank 2 and tank 3. The derivative of the sliding surface $\$$ s_ $1 \$$ can be written as follows:

$$
\dot{s}_{1}=l_{1}+b_{12} u_{2}
$$

with:

$$
l_{1}=\left(c_{3} \sqrt{\left|x_{3}-x_{2}\right|}-B_{4} \sqrt{x_{2}}-\dot{x}_{2 d}\right) \text { and } b_{12}=\frac{\lambda_{1}}{a}
$$

Similarly, the derivative of $s_{2}$ is:

$$
\dot{s}_{2}=l_{2}+b_{21} u_{1}+b_{22} u_{2}
$$

with:

$$
l_{2}=\lambda_{2}\left(c_{1} \sqrt{\left|x_{1}-x_{3}\right|}-c_{3} \sqrt{\left|x_{3}-x_{2}\right|}-\dot{x}_{3 d}\right)+c_{1} \frac{-2 c_{1} \sqrt{\left|x_{1}-x_{3}\right|}+c_{3} \sqrt{\left|x_{3}-x_{2}\right|}}{2 \sqrt{\left|x_{1}-x_{3}\right|}}-c_{3} \frac{c_{1} \sqrt{\left|x_{1}-x_{3}\right|}-2 c_{3} \sqrt{\left|x_{3}-x_{2}\right|}+B_{4} \sqrt{x_{2}}}{2 \sqrt{\left|x_{3}-x_{2}\right|}}-\dot{x}_{3 d}
$$

$$
b_{22}=c_{3} \frac{1}{2 a \sqrt{\left|x_{3}-x_{2}\right|}} \text { and } b_{21}=c_{1} \frac{1}{2 a \sqrt{\left|x_{3}-x_{1}\right|}}
$$

then

$$
\dot{s}=l+b u
$$

with:

$$
l=\left[\begin{array}{l}
l_{1} \\
l_{2}
\end{array}\right] \text { and } b=\left[\begin{array}{cc}
0 & b_{12} \\
b_{21} & b_{22}
\end{array}\right]
$$

The Control SMC with sat vector is:

$$
u=-b^{-1}\left(l+\left[\begin{array}{c}
k_{1} \operatorname{sign}\left(s_{1}\right) \\
k_{2} \operatorname{sign}(s)_{2}
\end{array}\right]\right)
$$

with:

$$
b^{-1}=\left[\begin{array}{cc}
-\frac{a c_{3} \sqrt{\left|x_{1}-x_{3}\right|}}{\lambda_{1} c_{1} \sqrt{\left|x_{3}-x_{2}\right|}} & \frac{2 a \sqrt{\left|x_{1}-x_{3}\right|}}{c_{1}} \\
\frac{a}{\lambda_{1}} & 0
\end{array}\right]
$$

The Control SMC with integrator vector is defined by:

$$
u=-b^{-1}\left(l+\left[\begin{array}{l}
k_{1} \operatorname{sign}\left(s_{1}\right) \\
k_{2} \operatorname{sign}\left(s_{2}\right)
\end{array}\right]\right)+K\left[\begin{array}{l}
\int\left(y_{1}-y_{1}^{d}\right) d t \\
\int\left(y_{2}-y_{2}^{d}\right) d t
\end{array}\right]
$$

with:

$$
K=\left[\begin{array}{ll}
K_{11} & K_{12} \\
K_{21} & K_{22}
\end{array}\right]
$$

\section{Simulation Results}

The controllers designed in Section 3 are simulated using the MATLAB software. The parameters of the three tank system Figure 1 are given in Table 1.The parameters for the both controls for three tanks are $\lambda=0.6, k_{1}=0.699$, $k_{2}=0.53, K_{11}=10^{-4}, K_{12}=18.10^{-3}, K_{21}=7.10^{-4}$ and $K_{22}$ $=10^{-4}$.

Simulation results are shown in Figures 2 to 4 . We can notice that in the absence of chattering in controls $u_{1}$ and $u_{2}$ both controls proposed and both output $h_{2}$ and $h_{3}$ follows their desired references $h_{2 d}$ and $h_{3 d}$.

However, when we inject a disturbance at $\mathrm{t}=1500 \mathrm{~s}$ in the outflow pipes of tank 2 and tank 3, the tow controllers ensure the convergence of the water levels $h_{3}$ and $h_{2}$ to their desired references $h_{2 d}$ and $h_{3 d}$. We see in the Figure 3 when we add integral action, the steady state error is almost eliminated. This is the advantage of the controls proposed in multi-variables coupled system case. 


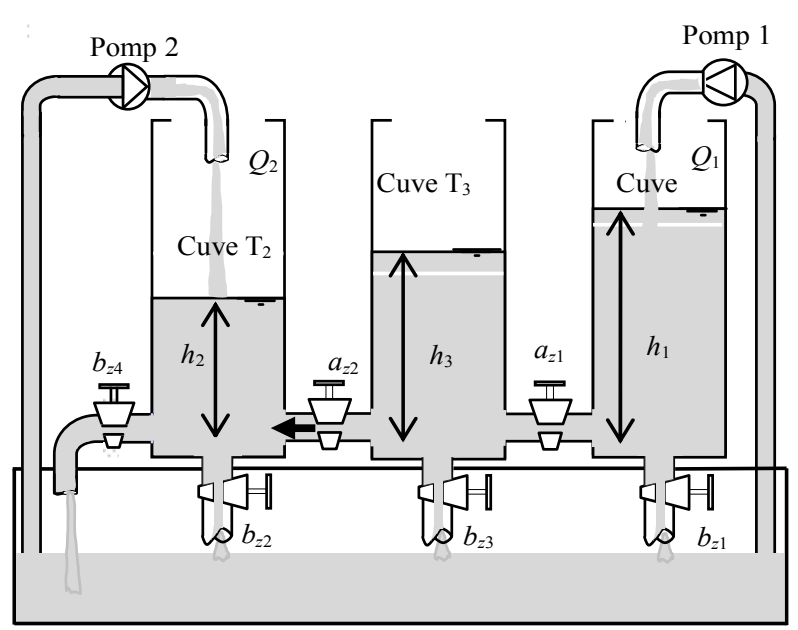

Figure 1. Three tank system.

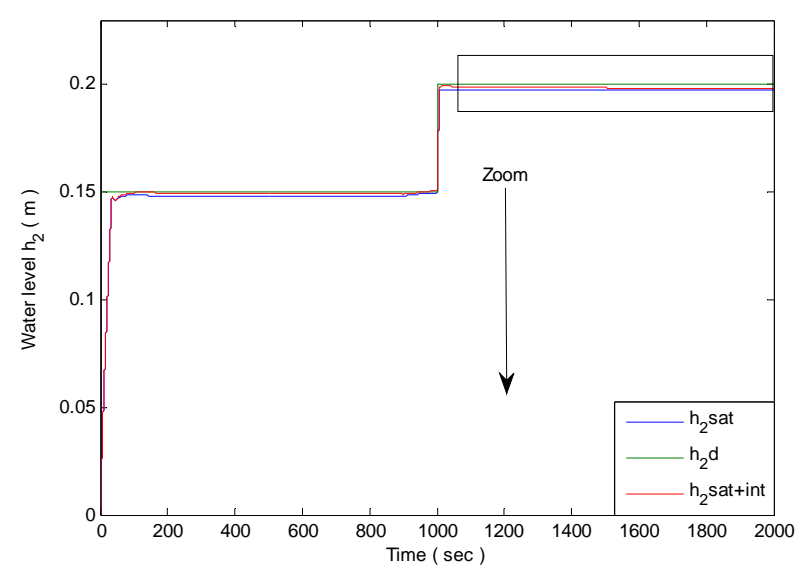

Figure 2. Liquid level in tank 2.

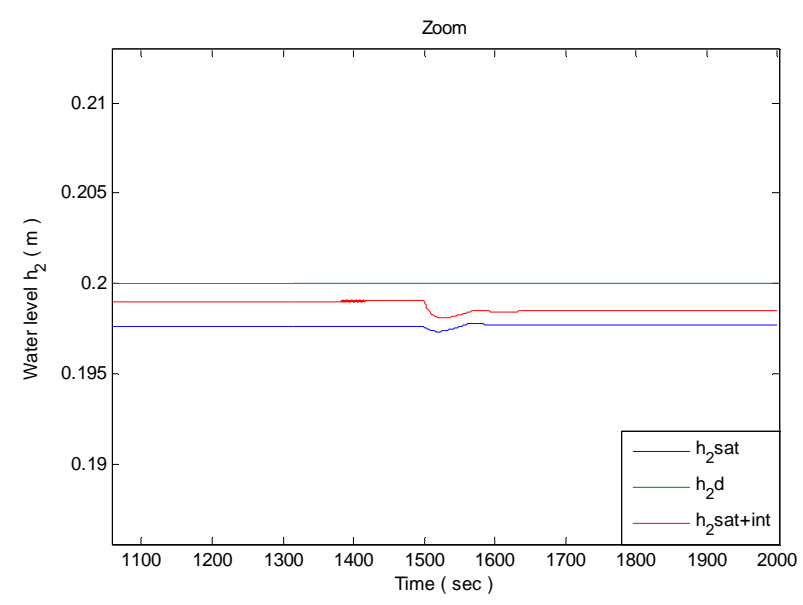

Figure 3. Liquid level with zoom in tank 2.

\section{Experimental Results}

The proposed control algorithms were tested on the physical laboratory plant (Figure 8) consisting of inter- connected three tank system. The objective is to control the liquid level of tanks 2 and 3. The experimental schemes have been done under Matlab/Simulink, using Real-Time Interface, and run on the DS1102 DSPACE system, which is equipped by a power $\mathrm{PC}$ processor. The control algorithm is implemented on DSP (TMS 320C31).

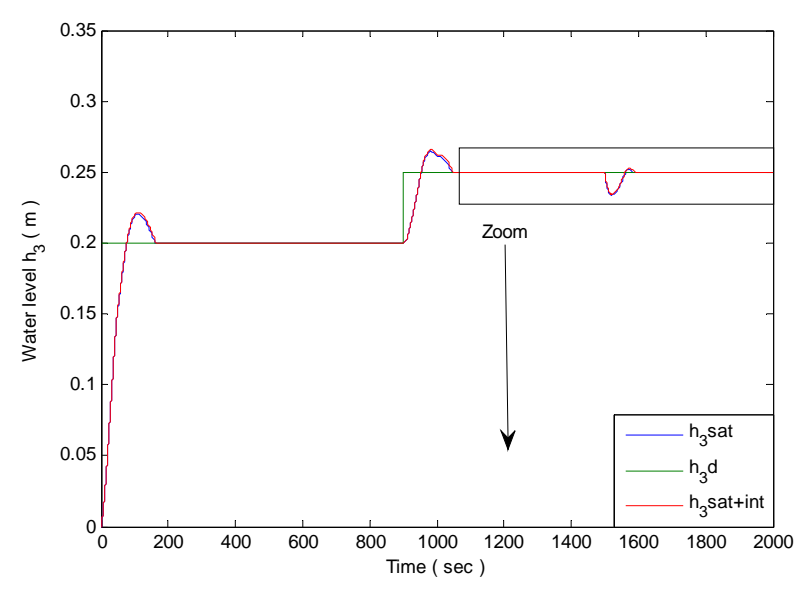

Figure 4. Liquid level in tank 3.

Table 1. Numerical values for physical parameters of the three tank system.

\begin{tabular}{|c|c|c|}
\hline Symbol & Value & Meaning \\
\hline$a$ & $0.0154 \mathrm{~m}^{2}$ & tank section \\
\hline$S_{n}$ & $2.510^{-5} \mathrm{~m}^{2}$ & cross-section of valve \\
\hline$a_{Z i}$ & $0 \leq a_{z i} \leq 1$ & flow correction term $(i=1,2,3)$ \\
\hline$b_{z i}$ & $0 \leq b_{z i} \leq 1$ & $\begin{array}{l}\text { leakage flow correction term } \\
\qquad(i=1,2,3)\end{array}$ \\
\hline$g$ & $9.81 \mathrm{~m} / \mathrm{s}^{2}$ & gravity constant $\mathrm{m} / \mathrm{s}^{2}$ \\
\hline$h_{\max }$ & $0.6 \mathrm{~m}$ & $\begin{array}{l}\text { maximum water level in each tank } \\
\qquad(i=1,2,3)\end{array}$ \\
\hline$Q_{\text {max }}$ & $1.1710^{-4} \mathrm{~m}^{3} / \mathrm{s}$ & $\begin{array}{l}\text { maximum inflow through pump i } \\
\qquad(i=1,2)\end{array}$ \\
\hline
\end{tabular}

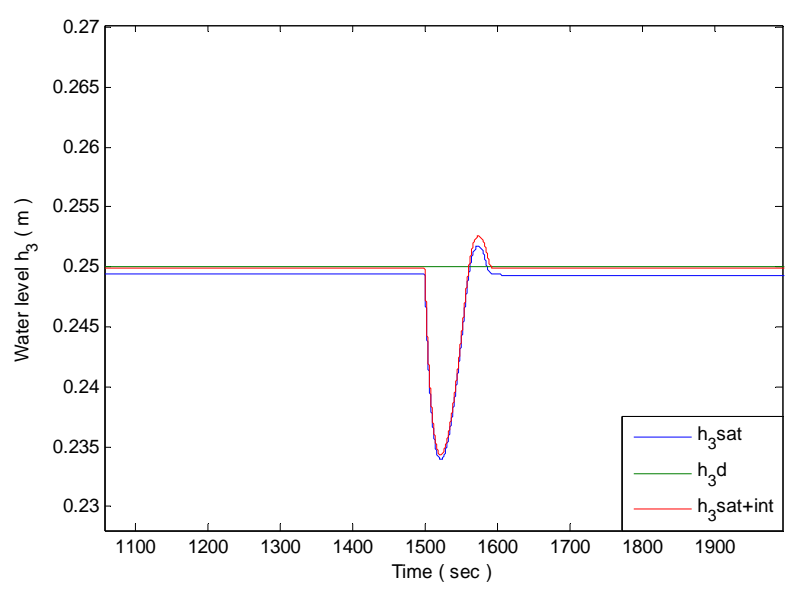

Figure 5. Liquid level with zoom in tank 3. 


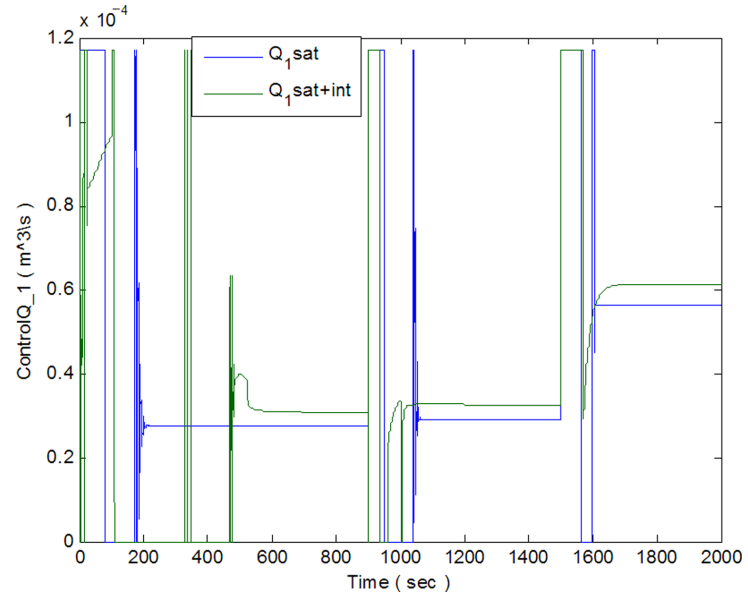

Figure 6. Input signals of control $Q_{1}$.

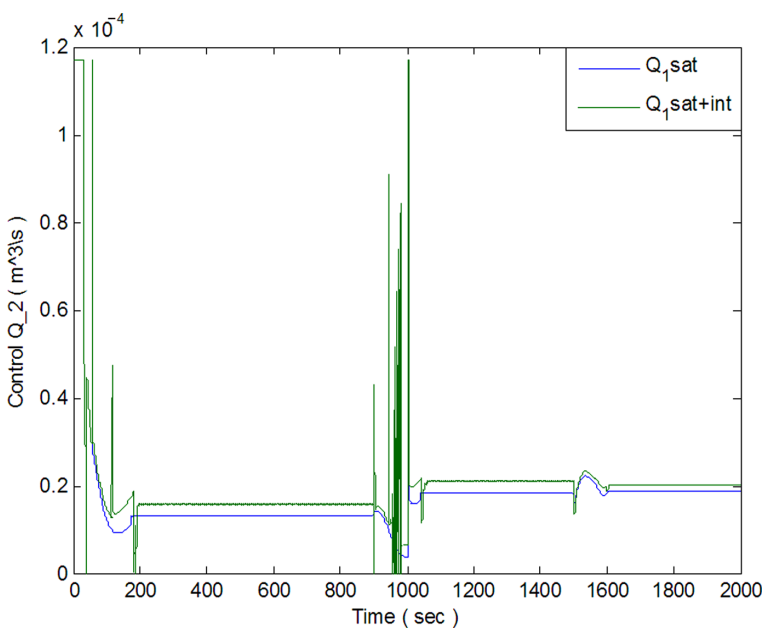

Figure 7. Input signals of control $Q_{2}$.

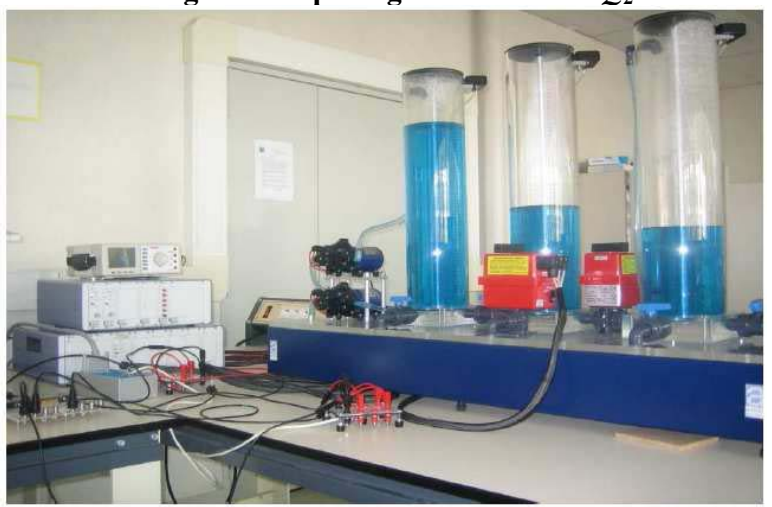

Figure 8. Real system. The parameters for both controls for three tanks are: $\lambda=0.6, k_{1}=0.46, k_{2}=0.32, K_{11}=10^{-3}, K_{12}$ $=3.10^{-4}, K_{21}=11.10^{-5}$ and $K_{22}=7.10^{-4}$.

For given references we remark that water levels $h_{2 d}$ and $h_{3 d}$ reach their references without overshooting. When we change the references we obtain the same response. In order to test the robustness of our strategy with respect to parameter uncertainties and disturbances,

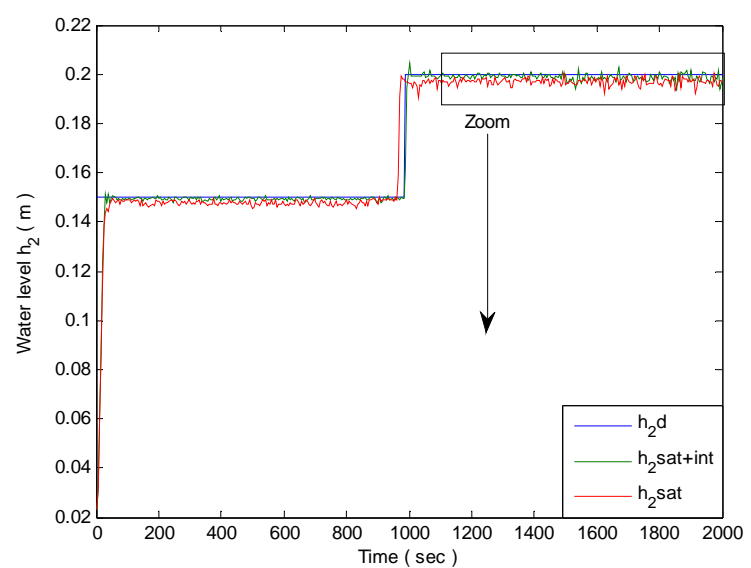

Figure 9. Liquid level in tank 2.

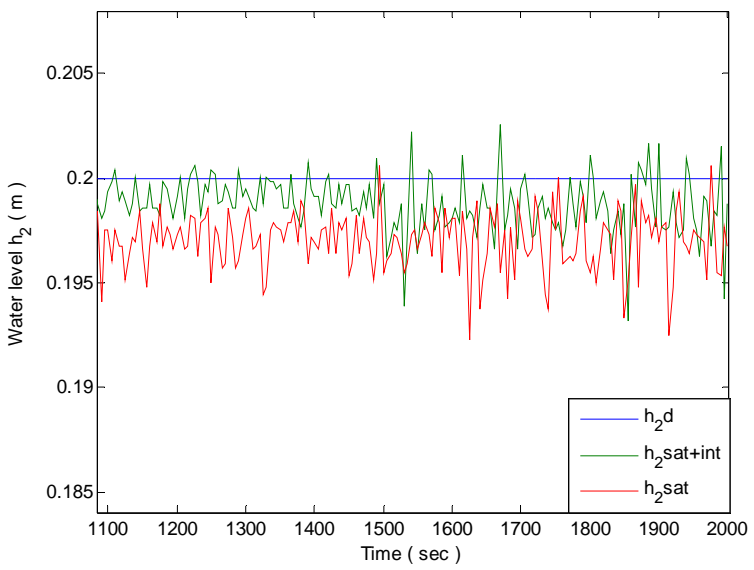

Figure 10. Liquid level with zoom in tank 2.

we varied the parameters $c_{1}$ and $c_{3}$ by closing and opening a little bit the valves $a_{z 1}$ and $a_{z 2}$ and we introduce a permanent leakage in the outflow pipes of tank 2 and tank 3 at $\mathrm{t}=1500 \mathrm{~s}$. We remark at $1500 \mathrm{~s}$ in the outflow pipes of tank 2 and tank 3 , the two controllers ensure the convergence of the water level $h_{3}$ and $h_{2}$ to their desired references $h_{3 d}$ and $h_{2 d}$ (Figures 9 and 11).

Then, the advantage of the sliding mode control with integrator in simulation and experimental results is the attenuation of error static (Figures 3, 5, 10, and 12).

Moreov er, we can also observe that control inputs $Q_{1}$ and $Q_{2}$ are smooth and the chattering phenomenon is almost eliminated (Figures 6, 7, 13 and 14).

\section{Conclusion}

In this paper, robust sliding mode control for a class of MIMO nonlinear systems was presented. In order to eliminate chattering phenomenon and the steady state error induced by the use of sat function, continuous sliding mode control, combined with a conditional integrator 


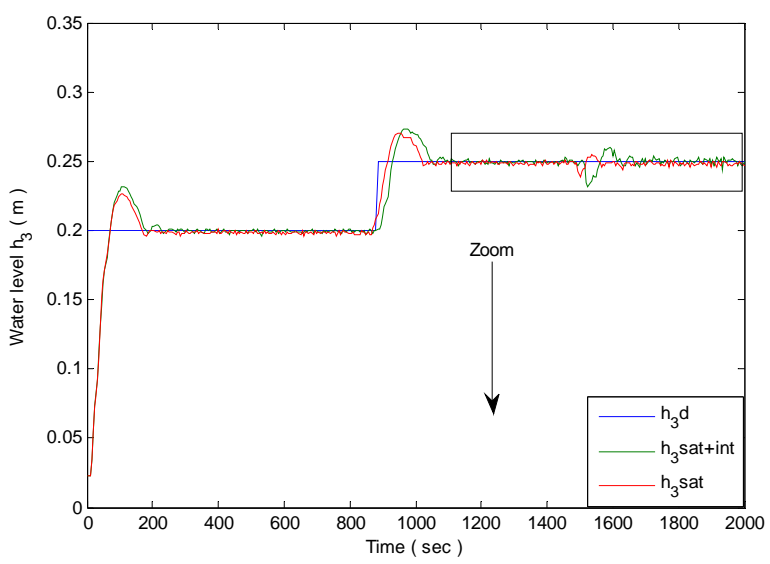

Figure 11. Liquid level in tank 3.

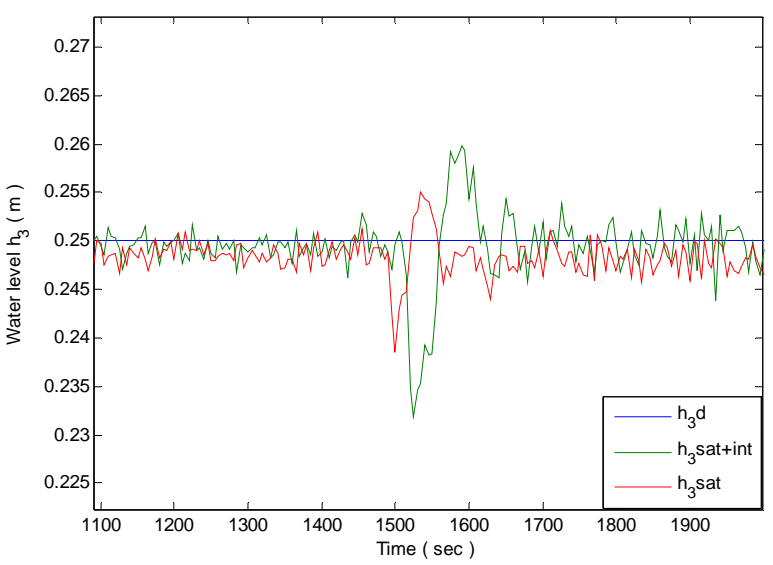

Figure 12. Liquid level with zoom in tank 3.

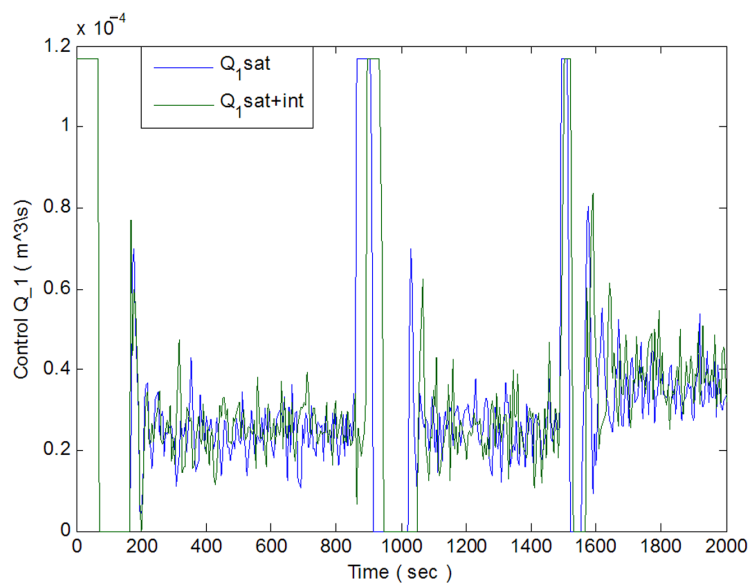

Figure 13. Input signals of control $Q_{1}$

admittance coefficients of various pipes, leakage in the tanks and uncertainty due to neglected pump dynamics. was proposed. This control was applied to the levels control of MIMO nonlinear three tanks system benchmark. The simulation and experimental results show robustness to parameter variations such as tank Section,

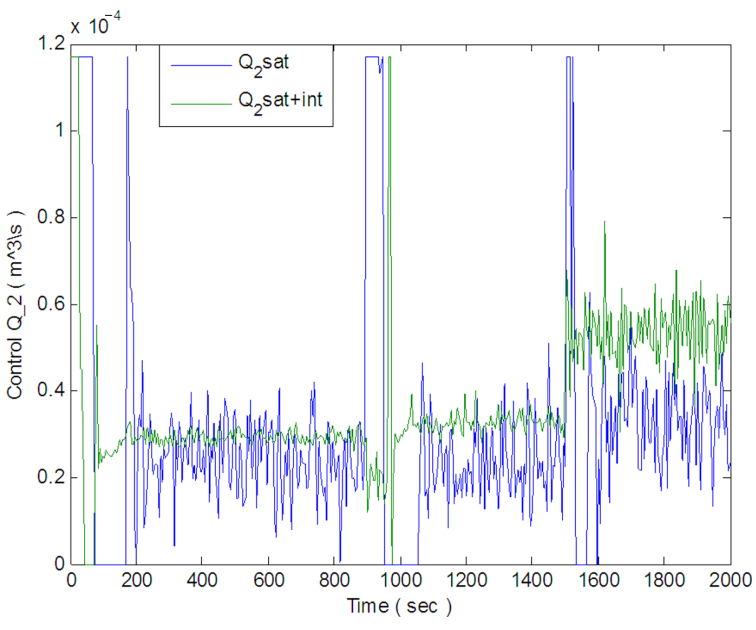

Figure 14. Input signals of control $Q_{2}$.

The simulation and experimental results, compared with those obtained without integrator, confirm the effectiveness of our control strategy.

\section{References}

[1] V. Utkin, "Sliding Mode in Control and Optimization," Springer-Verlag, Berlin, 1992

[2] V. Utkin, J. Guldner and J. Shi, "Sliding Modes Control in Electromechanical Systems," CRC press, Boca Raton, 1999.

[3] L. Fridman, "An Averaging Approach to Chattering," IEEE Transactions on Automatic Control, Vol. 46, No. 8, 2001, pp. 1260-1265. doi:10.1109/9.940930

[4] H. Abid, M. Chtourou and A. Toumi, "Robust Fuzzy Sliding Mode Controller for Discrete Nonlinear Systems," International Journal of Computers, Communications and Control, Vol. 3, No. 1, 2008, pp. 6-20.

[5] S. Mahieddine-Mahmoud, L. Chrifi-Alaoui, V. van Assche, J.-M. Castelain and P. Bussy, "Sliding Mode Control Based on Field Orientation for an Induction Motor," IEEE Industrial Electronics Society, IECON, North Carolina, 6-20 November 2005, pp. 181-186.

[6] H. Hashimoto, H. Yamamoto, S. Yanagisawa and F. Harashima, "Brushless Servo Motor Control Using Variable Structure Approach," IEEE Transactions on Industry Applications, Vol. 24, No. 1, 1988, pp. 160-170. doi: $10.1109 / 28.87267$

[7] I. Eker and S. A. Akinal, "Sliding Mode Control with Integral Augmented Sliding Surface: Design and Experimental Application to an Electromechanical System," Electrical Engineering, Vol. 90, No. 3, 2008, pp. 189-197. doi:10.1007/s00202-007-0073-3

[8] A. Levant, "Chattering Analysis," IEEE Transactions on Automatic Control, Vol. 55, No. 6, 2010, pp. 1380-1389. doi:10.1109/TAC.2010.2041973

[9] C. Edwards and S. Spurgeon, "Sliding Mode Control: Theory and Applications," Taylor-Francis, London, 1998.

[10] J. J. E. Slotine and W. Li, "Applied Nonlinear Control," 
Prentice-Hall International, New Jersey, 1991.

[11] I. Eker snd S. A. Akinal, "Sliding Mode Control with Integral Action and Experimental Application to an Electromechanical System Application to an Electromechanical System," IEEE-ICSC Congress on Computational Intelligence Methods and Applications, Istanbul, 2-14 December 2005, p. 6. doi:10.1109/CIMA.2005.1662303

[12] S. Seshagiri and H. K. Khalil, "Robust Output Feedback Regulation of Minimum-Phase Nonlinear Systems Using Conditional Integrators," Automatic, Vol. 41, No. 1, 2005, pp. 43-54. doi:10.1016/j.automatica.2004.08.013

[13] R. Benayache, L. Chrifi-Alaoui, P. Bussy and J. M. Cas- telain, "Sliding Mode Control with Integral Corrector: Design and Experimental Application to an Interconnected System," Mediterranean Conference on Control and Automation, Thessaloniki, 24-26 June 2009, pp. 831-836. doi:10.1109/MED.2009.5164647

[14] T. Floquet, S. K. Spurgeon and C. Edwards, "An Output Feedback Sliding Mode Control Strategy for MIMO Systems of Arbitrary Relative Degree," International Journal of Robust and Nonlinear Control, Vol. 21, No. 2, 2010, pp. 119-133. doi:10.1002/rnc.1579

[15] K. Ogata, "System Dynamics," Englewood Cliffs, New Jersey, 1978. 